\title{
Influence of Uniaxial Stress on the Indirect Absorption Edge in Silicon and Germanium
}

\section{Balslev, I.}

\section{Published in:}

PHYSICAL REVIEW

Link to article, DOI:

10.1103/PhysRev.143.636

Publication date:

1966

Document Version

Publisher's PDF, also known as Version of record

Link back to DTU Orbit

Citation (APA):

Balslev, I. (1966). Influence of Uniaxial Stress on the Indirect Absorption Edge in Silicon and Germanium. PHYSICAL REVIEW, 143(2), 636-647. https://doi.org/10.1103/PhysRev.143.636

\section{General rights}

Copyright and moral rights for the publications made accessible in the public portal are retained by the authors and/or other copyright owners and it is a condition of accessing publications that users recognise and abide by the legal requirements associated with these rights.

- Users may download and print one copy of any publication from the public portal for the purpose of private study or research.

- You may not further distribute the material or use it for any profit-making activity or commercial gain

- You may freely distribute the URL identifying the publication in the public portal

If you believe that this document breaches copyright please contact us providing details, and we will remove access to the work immediately and investigate your claim. 
As an independent check of the position of the upper Co energy level, the resistivity of several Co-compensated $n$-type samples has been measured. For these measurements samples were chosen which had resistivities of 7,8 , and $22 \Omega \mathrm{cm}$ before compensation and less than $20000 \Omega \mathrm{cm}$ after compensation. Hall mobilities of these samples were greater than $1000 \mathrm{~cm}^{2} /$ volt sec after compensation. These measurements indicate a zero-temperature thermal ionization energy ${ }^{9}$ from the upper Co level to the conduction band of $0.55 \pm 0.02 \mathrm{eV}$.

\section{DISCUSSION OF ENERGY LEVELS}

We have obtained three independent measurements of the energy of the upper Co level in $\mathrm{Si}$, two with respect to the valence band edge, one with respect to the conduction band edge. As the measurements have been performed at different temperatures, and the energy gap of $\mathrm{Si}$ varies with temperature, ${ }^{10}$ we have adjusted all values to room temperature assuming that the impurity level changes in proportion with the energy gap. Based on a room temperature gap of 1.11 $\mathrm{eV},,^{10}$ we find the position of the upper Co acceptor level

${ }^{9}$ G. L. Pearson and J. Bardeen, Phys. Rev. 75, 865 (1949).

${ }^{10}$ G. G. Macfarlane, T. P. McLean, J. E. Quarington, and V. Roberts, Phys. Rev. 111, 1245 (1958). below the conduction band to be $0.52 \pm 0.02 \mathrm{eV}$ from negative photoconductivity; $0.54 \pm 0.02 \mathrm{eV}$ from positive photoconductivity; and $0.53 \pm 0.02 \mathrm{eV}$ from resistivity versus temperature

For the lower Co acceptor level at room temperature an energy of $0.35 \pm 0.02 \mathrm{eV}$ from the valence-band edge is indicated from negative photoconductivity measurements.

\section{SUMMARY AND CONCLUSIONS}

We have demonstrated that Co introduces two deep acceptor levels in $\mathrm{Si}$, and moreover produces negative photoconductivity consistent with the model proposed by Stockmann ${ }^{5}$ and by Johnson and Levinstein ${ }^{6}$ for similar effects in Ge. The two acceptor levels are found to be $0.35 \pm 0.02 \mathrm{eV}$ above the top of the valence band, and $0.53 \pm 0.02 \mathrm{eV}$ below the bottom of the conduction band, respectively.

\section{ACKNOWLEDGMENTS}

We wish to thank J. Bardeen, D. Aspnes, M. D. Sirkis, and F. Germano for some useful discussions, and G. D. Clark for help in performing some measurements. We would also like to thank C. T. Sah for the use of some of his equipment.

\title{
Influence of Uniaxial Stress on the Indirect Absorption Edge in Silicon and Germanium
}

\author{
I. BALSLEV \\ Physics Laboratory III, Technical University of Denmark, Lyngby, Denmark
}

(Received 20 September 1965)

\begin{abstract}
The indirect optical absorption edge in silicon and germanium has been studied in the presence of shear strain. The splitting observed in the transmission spectrum is dependent on the direction and magnitude of the applied stress and on the polarization of the light with respect to the stress axis. The results have been interpreted in terms of changes in the valence- and conduction-band structure with strain. Neglecting strain dependence of phonon energies, various deformation potential constants have been determined from the experiments. The values are: $\mathrm{Si}, 80^{\circ} \mathrm{K}: \Xi_{u}=8.6 \pm 0.2 \mathrm{eV},|b|=2.4 \pm 0.2 \mathrm{eV},|d|=5.3 \pm 0.4 \mathrm{eV}, \Xi_{d}+\frac{1}{3} \Xi_{u}$ $-a=3.8 \pm 0.5 \mathrm{eV} . \mathrm{Si}, 295^{\circ} \mathrm{K}: \Xi_{u}=9.2 \pm 0.3 \mathrm{eV},|b|=2.2 \pm 0.3 \mathrm{eV}, \Xi_{d}+\frac{1}{3} \Xi_{u}-a=3.1 \pm 0.5 \mathrm{eV} . \mathrm{Ge}, 80^{\circ} \mathrm{K}:$ $\Xi_{u}=16.2 \pm 0.4 \mathrm{eV}, b=-1.8 \pm 0.3 \mathrm{eV}, d=-3.7 \pm 0.4 \mathrm{eV}, \Xi_{d}+\frac{1}{3} \Xi_{u}-a=-2.0 \pm 0.5 \mathrm{eV}$. An observed nonlinear dependence of the splitting on stress has been interpreted as shifts of the exciton energies with uniaxial stress. A special experimental technique using a vibrating slit in the spectrometer was used in order to obtain an accurate determination of the fine structure in the absorption spectrum.
\end{abstract}

\section{INTRODUCTION}

$\mathbf{I}^{\mathrm{N}}$ $\mathrm{N}$ the presence of shear strain in a cubic semiconductor the reduced degree of symmetry gives rise to significant changes in the band structure. Based on group theory and $\mathbf{k} \cdot \mathbf{p}$ perturbation calculations, several theoretical investigations ${ }^{1-5}$ have been carried

${ }^{1}$ C. Herring and E. Vogt, Phys. Rev. 101, 944 (1956).

2 R.W. Keyes, Solid State Phys. 11, 150 (1961).

${ }^{3}$ G. E. Pikus and G. L. Bir, Fiz. Tverd. Tela 1, 1642 (1959)

[English Transl.: Soviet Phys.-Solid State 1, 1502 (1959)].

${ }^{4}$ W. H. Kleiner and L. M. Roth, Phys. Rev. Letters 2, 334 (1959).

${ }^{5}$ H. Hasegawa, Phys. Rev. 129, 1029 (1963). out on the valence and conduction bands in $\mathrm{Si}$ and Ge.

Thus, the effect of shear strain on the degenerate valence-band edge is found to be a lifting of the degeneracy at $\mathbf{k}=0$. The constant-energy surfaces being "warped" or "fluted" in the unstrained crystal develop into ellipsoidal forms. This behavior can be described by introduction of the deformation potentials $a, b$, and $d$ defined by Pikus and Bir. ${ }^{3}$

For the conduction-band minima off the center of the Brillouin zone the shapes of the energy surfaces are unchanged to the first order in stress whereas the extremum energy of a particular valley will depend on 
TABLE I. Conduction-band splitting $\Delta E_{c}{ }^{i}-\Delta E_{c}{ }^{0}$ for uniaxially stressed $\mathrm{Si}$ and Ge. $T$ is the stress (positive for tensile stress), $\Xi_{u}$ is the deformation potential, and $S_{11}, S_{12}$, and $S_{44}$ are compliance coefficients.

\begin{tabular}{|c|c|c|c|c|}
\hline $\begin{array}{c}\text { Material } \\
\text { Stress direction }\end{array}$ & Valley direction & $\Delta E_{c}^{i}-\Delta E_{c}^{0}$ & Valley direction & $\Delta E_{c}{ }^{i}-\Delta E_{c}^{0}$ \\
\hline$[001]$ & $\begin{array}{ll}{[001]} & {[00 \overline{1}]} \\
{[100]} & {[010]} \\
{[\overline{100}]} & {[0 \overline{1} 0]}\end{array}$ & $\begin{array}{r}\frac{2}{3}\left(S_{11}-S_{12}\right) \Xi_{u} T \\
-\frac{1}{3}\left(S_{11}-S_{12}\right) \Xi_{u} T\end{array}$ & Any & 0 \\
\hline [111] & Any & 0 & $\begin{array}{c}[111]] \\
{[11 \overline{1}][1 \overline{1} 1]} \\
{[\overline{1} 11]}\end{array}$ & $\begin{array}{r}\frac{1}{3} S_{44} \Xi_{u} T \\
-\frac{1}{9} S_{44} \Xi_{u} T\end{array}$ \\
\hline$[110]$ & 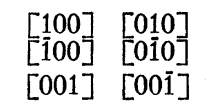 & $\begin{array}{r}\frac{1}{6}\left(S_{11}-S_{12}\right) \Xi_{u} T \\
-\frac{1}{3}\left(S_{11}-S_{12}\right) \Xi_{u} T\end{array}$ & $\begin{array}{ll}{[111]} & {[11 \overline{1}]} \\
{[\overline{1} 11]} & {[1 \overline{1} 1]}\end{array}$ & $\begin{array}{r}\frac{1}{6} S_{44} \Xi_{u} T \\
-\frac{1}{6} S_{44} \Xi_{u} T\end{array}$ \\
\hline
\end{tabular}

the magnitude of the stress and its direction with respect to the $k$ vector of the valley. These shifts are described by the deformation potentials $\Xi_{d}$ and $\Xi_{u}$ of Herring and Vogt. ${ }^{1}$

Experimentally, intrinsic properties and free-carrier effects in strained $\mathrm{Si}$ and Ge have been studied by electrical, ${ }^{6-8}$ optical, ${ }^{9,10}$ and microwave ${ }^{11}$ techniques. The results of these measurements seem to verify the above theories, but the magnitudes of the deformation potentials obtained by the different methods deviate more than it seems possible to ascribe to experimental uncertainties.

In this paper we shall describe an independent experimental method for determining the deformation potential constants based on an analysis of the change in the indirect absorption edge due to uniaxial stress.

The structure of the indirect absorption edge in strain-free silicon and germanium crystals has been discussed in detail by several authors. ${ }^{12,13}$

This structure is characterized by certain threshold energies corresponding to the creation of an electronhole pair with simultaneous emission or absorption of a phonon in order to fulfill the condition of momentum conservation.

Owing to the lifting of the energy degeneracy with shear strain of the valence- and conduction-band edges these thresholds will split up. An analysis of the fine structure of the thresholds and its dependence on direction and magnitude of uniaxial stress makes it

${ }^{6}$ F. J. Morin, T. H. Geballe, and C. Herring, Phys. Rev. 101, 525 (1957).

${ }^{7}$ J. E. Aubrey, W. Gubler, T. Henningsen, and S. H. Koenig, Phys. Rev. 130, 1667 (1963).

${ }^{8}$ G. L. Bir, A. I. Bloom, and U. V. Ilisavsky, Proceedings of the Seventh International Conference on the Physics of Semiconductors, Paris, 1964 (Dunod Cie., Paris, 1965), p. 529.

${ }^{9}$ A. M. Glass, Can. J. Phys. 43, 12 (1965).

${ }_{10} \mathrm{~K}$. J. Schmidt-Tiedemann, Proceedings of the Sixth International Conference on the Physics of Semiconductors, Exeter, 1962 (Institute of Physics and the Physical Society, London, 1962), p. 191.

${ }_{11}^{11}$ J. C. Hensel and G. Feher, Phys. Rev. 129, 1041 (1963).

12 G. G. Macfarlane, T. P. McLean, J. E. Quarrington, and V. Roberts, Phys. Rev. 108, 1377 (1957); hereafter referred to as MMQR.

${ }^{13}$ R. J. Elliott, Phys. Rev. 108, 1384 (1957). possible to determine the deformation potentials of the two band edges.

\section{BAND STRUCTURE OF STRAINED SILICON AND GERMANIUM}

The energy degeneracy in a many-valley cubic semiconductor is in general lifted by shear strain. Herring and $\operatorname{Vogt}^{1}$ have applied symmetry arguments in the studies of this phenomena and found that the energy shift $\Delta E_{c}{ }^{i}$ of valley $i$ for an arbitrary homogeneous deformation described by the second-rank strain tensor $\varepsilon$ can be expressed as

$$
\Delta E_{c}{ }^{i}=\left[\Xi_{d} 1+\Xi_{u}\left\{\mathbf{a}_{i} \mathbf{a}_{i}\right\}\right]: \varepsilon,
$$

where $\mathbf{1}$ is the unit tensor, $\mathbf{a}_{i}$ is a unit vector parallel to the $\mathbf{k}$ vector of valley $i$, and \{\} denotes a dyadic product. The shift of the mean energy of the band extrema is

$$
\Delta E_{c}^{0}=\left(\Xi_{d}+\frac{1}{3} \Xi_{u}\right) 1: \varepsilon
$$

and the quantity $\Delta E_{c}{ }^{i}-\Delta E_{c}{ }^{0}$ is given in Table I for $\mathrm{Si}$ and $\mathrm{Ge}$ in case of uniaxial stress parallel to the crystal directions [001], [111], and [110].

Without strain and spin-orbit coupling the valenceband edge in silicon and germanium is a sixfold degenerate $p$ multiplet with symmetry $\Gamma_{25}{ }^{\prime}$. The spin-orbit coupling partially lifts the degeneracy into a fourfold $p_{3 / 2}$ multiplet and a $p_{1 / 2}$ doublet with a separation $\Lambda=0.044 \mathrm{eV}$ in Si and $\Lambda=0.3 \mathrm{eV}$ in Ge. The edge, the upper $J=\frac{3}{2}$ state, is for $\mathbf{k} \neq 0$ a pair of Kramers doublets designated as "light" and "heavy" holes. $\mathbf{k} \cdot \mathbf{p}$ perturbation applied to this edge yields energy surfaces of the form $^{14}$

$$
E(\mathbf{k}) \pm=A k^{2} \pm\left[B^{2} k^{4}+C^{2}\left(k_{x}{ }^{2} k_{y}{ }^{2}+k_{x}{ }^{2} k_{z}{ }^{2}+k_{y}{ }^{2} k_{z}{ }^{2}\right)\right]^{1 / 2} \text {, }
$$

where $A, B$, and $C$ are inverse mass parameters related to the elements of the Shockley matrix ${ }^{14}$ by

$$
\begin{aligned}
A & =\frac{1}{3}(L+2 M)+\left(\hbar^{2} / 2 m_{0}\right), \\
B & =\frac{1}{3}(L-M), \\
C^{2} & =\frac{1}{3}\left(N^{2}-(L-M)^{2}\right) .
\end{aligned}
$$

${ }^{14}$ G. Dresselhaus, A. F. Kip, and C. Kittel, Phys. Rev. 98, 368 (1955). 
As pointed out by Pikus and $\mathrm{Bir}^{3}$, the degeneracy at $\mathbf{k}=0$ is lifted by shear strain. Neglecting the spin-orbit split-off band, they found that the band structure near $\mathbf{k}=\mathbf{0}$ for the strain $\boldsymbol{\varepsilon}$ is given by

$$
E(\mathbf{k})^{ \pm}=A k^{2}+a \mathbf{1}: \varepsilon \pm\left[E_{k k}{ }^{2}+E_{\epsilon k}{ }^{2}+E_{\epsilon \epsilon}{ }^{2}\right]^{1 / 2},
$$

where

$$
\begin{aligned}
E_{k k}{ }^{2} & =B^{2} k^{4}+C^{2}\left(k_{x}{ }^{2} k_{y}{ }^{2}+k_{x}{ }^{2} k_{z}{ }^{2}+k_{y}{ }^{2} k_{z}{ }^{2}\right), \\
E_{\epsilon \epsilon}{ }^{2} & =\frac{1}{2} b^{2}\left[\left(\epsilon_{x x}-\epsilon_{y y}\right)^{2}+\text { c.p. }\right]+d^{2}\left[\epsilon_{x y}{ }^{2}+\text { c.p. }\right), \\
E_{\epsilon}{ }^{2} & =B b\left[3\left(k_{x}{ }^{2} \epsilon_{x x}+\text { c.p. }\right)-k^{2} 1: \varepsilon\right] \\
& \quad+(2 / \sqrt{3}) N d\left[k_{x} k_{y} \epsilon_{x y}+\text { c.p. }\right] .
\end{aligned}
$$

$N$ is the off-diagonal element of the Shockley matrix, ${ }^{14}$ $a, b$, and $d$ are deformation potentials, and c.p. stands for cyclic permutation with respect to the indices $x, y$, and $z$. It is seen that the band extrema are separated by the energy $2\left|E_{\epsilon \epsilon}\right|$ proportional to the strain. For sufficiently small values of $\mathbf{k}$ the surfaces of constant energy given by Eq. (2.5) are ellipsoids.

Hasegawa $^{5}$ has found, however, that the small spin-orbit splitting in $\mathrm{Si}, \Lambda=0.044 \mathrm{eV}$, gives rise to slightly nonlinear shifts of one of the band extrema within obtainable magnitudes of strain. The $6 \times 6$ Hamiltonian including the spin-off band has simple solutions for the stress directions [001] and [111] as indicated in Ref. 5. For $\mathbf{k}=0$ we find that Eq. (2.5) attains the form ${ }^{15}$

$$
\begin{aligned}
& E(0)^{+}=a 1: \varepsilon+\frac{1}{2}(\delta-\Lambda)+\frac{1}{2}\left[(\delta+\Lambda)^{2}+8 \delta^{2}\right]^{1 / 2}, \\
& E(0)^{-}=a 1: \varepsilon-\delta,
\end{aligned}
$$

where

$$
\begin{array}{lll}
\delta=+b\left(\epsilon_{z z}-\epsilon_{x x}\right) & \text { for } & \text { stress } \|[001], \\
\left.\delta=+2 / \sqrt{3} d \epsilon_{x y}\right) & \text { for } & \text { stress } \|[111] .
\end{array}
$$

The two cases in Eq. (2.6) correspond to total angular momentum quantum number $M_{J}= \pm \frac{1}{2}$ and $\pm \frac{3}{2}$, respectively $\left(M_{J}\right.$ quantized in the stress direction). Furthermore, it was shown ${ }^{5}$ that Eq. (2.6) holds for any direction of uniaxial stress if the quantity $\delta$ is stressisotropic.

Thus, for the indirect gap of strained silicon and germanium we obtain

$$
E_{g}{ }^{i j}=E_{g}{ }^{0}+\Delta E_{g}{ }^{0}+\left(\Delta E_{c}{ }^{i}-\Delta E_{c}{ }^{0}\right)+(-1)^{j}\left|E_{\epsilon \epsilon}\right|,
$$

where $i$ denotes the valley and $j=1,2$ corresponds to the valence bands. $E_{g}{ }^{0}$ is the energy gap of the strainfree crystal, and

$$
\begin{aligned}
\Delta E_{g}{ }^{0}=\left(\Xi_{d}+\frac{1}{3} \Xi_{u}-a\right) & 1: \varepsilon \\
& =\left(\Xi_{d}+\frac{1}{3} \Xi_{u}-a\right)\left(S_{11}+2 S_{12}\right) T
\end{aligned}
$$

is the mean shift of the gap for uniaxial stress $T$. The last two terms in Eq. (2.7) are listed in Tables I and II.

15 The figure 8 in this expression was incorrectly reported to be 2 in a previous publication by the author (Ref. 25).
TABLE II. Valence-band-splitting parameter $\left|E_{\epsilon \epsilon}\right|$ for uniaxial stress $T . b$ and $d$ are deformation potentials and $S_{11}, S_{12}$, and $S_{44}$ are compliance coefficients.

\begin{tabular}{cc}
\hline \hline Stress direction & $\left|E_{\mathrm{\epsilon \epsilon}}\right|$ \\
\hline$[001]$ & $\left|b\left(S_{11}-S_{12}\right) T\right|$ \\
{$[111]$} & $\left|\frac{d}{2 \sqrt{3}} S_{44} T\right|$ \\
{$[110]$} & $\frac{1}{2}\left[b^{2}\left(S_{11}-S_{12}\right)^{2}+3\left(\frac{d}{2 \sqrt{3}} S_{44}\right)^{2}\right]^{1 / 2}|T|$ \\
\hline
\end{tabular}

For high magnitudes of stress in $\mathrm{Si}$, the term $(-1)^{j}\left|E_{\epsilon \epsilon}\right|$ has to be modified according to the two cases in Eq. (2.6).

\section{PHONONS IN THE PRESENCE OF STRAIN}

The energy of the phonons participating in the indirect processes is in general dependent on strain. The energy shifts can be expressed by a formula similar to Eq. (2.1). The magnitudes of the deformation potentials associated with the phonon spectrum are not well known. It may be assumed, however, that the relative shift in the phonon energies due to strain is less than 5 times the magnitude of the strain, and in this case the phonon contribution to the splitting of the indirect thresholds can be neglected. This assumption was experimentally verified for the transverse optic (TO) phonon in Si in the present work by comparing the splitting of the two thresholds corresponding to emission and absorption of this phonon.

\section{INDIRECT EXCITONS IN STRAINED SILICON AND GERMANIUM}

As shown by Elliott, ${ }^{13}$ the spectral shape of the direct and indirect absorption edges can be explained by the presence of electron-hole interaction. His theory concerns simple spherical bands in which case the ground-state binding energy $E_{\text {ex }}$ of the exciton is given explicitly by the effective masses and the dielectric constant. For degenerate and anisotropic band edges McLean and Loudon ${ }^{16}$ have introduced a variational procedure for calculation of $E_{\text {ex }}$. The splitting obtained there in the ground-state level of the indirect excitons in $\mathrm{Si}$ and $\mathrm{Ge}$ has minor interest for our work as it was unresolved in the present measurements. The calculated mean values of $E_{\text {ex }}$ were $12.7 \mathrm{MeV}$ in $\mathrm{Si}$ and $3.0 \mathrm{MeV}$ in Ge.

When the strain-induced splitting of the valence bands is comparable to $E_{\mathrm{ex}}$, the theoretical method of Ref. 16 is inadequate, and more complicated procedures are required for computation of the exciton energies. In the case of completely decoupled valence bands the

${ }_{16}$ T. P. McLean and R. Loudon, J. Phys. Chem. Solids 13, 1 (1960). 


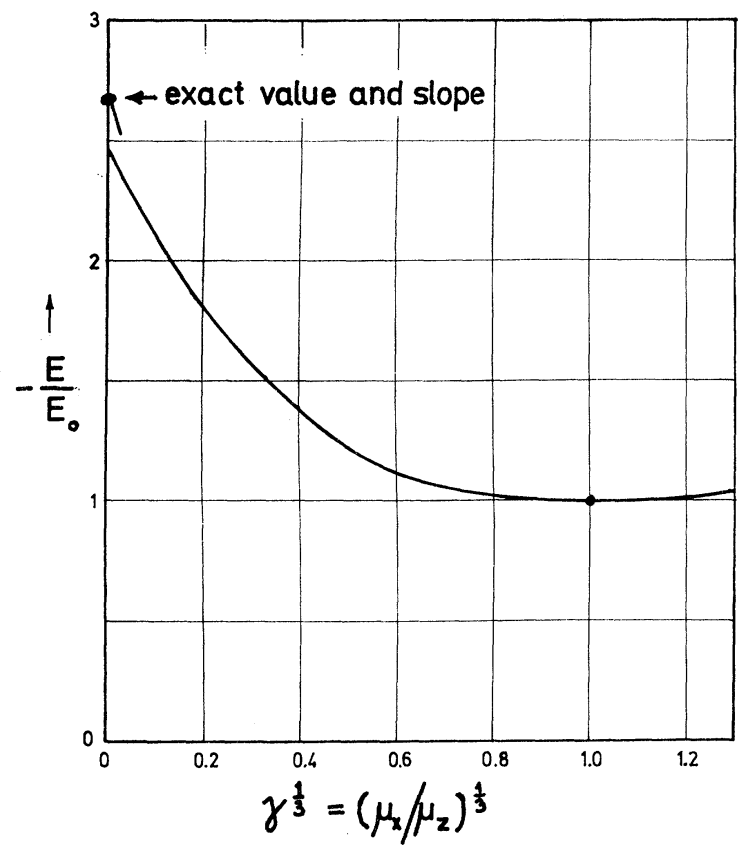

Fig. 1. Ground-state energy of the donor problem as function of the mass ratio $\gamma=\left(\mu_{x} / \mu_{z}\right)$ calculated by Kohn and Luttinger (Ref. 17). Here, the results are given in units of $E_{0}=3 e^{4} /\left[2 \hbar^{2} k^{2}\left(2 \mu_{x}{ }^{-1}+\mu_{z}{ }^{-1}\right)\right]$.

The analytic expression is given in Ref. 18 .

calculation of $\mathrm{E}_{\mathrm{ex}}$ should be based on two nondegenerate anisotropic bands for the electron and hole. The reciprocal mass tensor for the relative motion of the pair of particles is given by

$$
\mathbf{u}^{-1}=\mathbf{m}_{v}^{-1}+\mathbf{m}_{c}^{-1},
$$

where $\mathbf{m}_{v}^{-1}$ and $\mathbf{m}_{c}^{-1}$ are reciprocal mass tensors of the valence and conduction bands. In the coordinate system in which $\boldsymbol{u}^{-1}$ is diagonal $E_{\text {ex }}$ is the lowest eigenvalue of the equation

$$
\begin{array}{r}
{\left[-\frac{1}{2} \hbar^{2}\left(\frac{1}{\mu_{x}} \frac{\partial^{2}}{\partial x^{2}}+\frac{1}{\mu_{y}} \frac{\partial^{2}}{\partial y^{2}}+\frac{1}{\mu_{z}} \frac{\partial^{2}}{\partial z^{2}}\right)\right.} \\
\left.-\frac{e^{2}}{\kappa\left(x^{2}+y^{2}+z^{2}\right)^{1 / 2}}-E\right] \Phi(x, y, z)=0,
\end{array}
$$

where $\mu_{x}^{-1}, \mu_{y}^{-1}$, and $\mu_{z}^{-1}$ are the elements of the diagonalized $\boldsymbol{u}^{-1}$ tensor and $\kappa$ is the dielectric constant.

For $\mu_{x}=\mu_{y} \neq \mu_{z}$, Eq. (4.2) is similar to that of the donor problem investigated by Kohn and Luttinger. ${ }^{17}$ They calculated the approximate value of the groundstate energy $E$ of Eq. (4.2) as a function of the quantity

$$
\gamma \equiv \mu_{x} / \mu_{z}
$$

in the range $0<\gamma<1$.

${ }^{17}$ W. Kohn and J. M. Luttinger, Phys. Rev. 98, 915 (1955).
Further investigations for $\gamma>1$ are reported by Keyes. ${ }^{18}$ The ground-state energy $E$ in units of

$$
E_{0}=\frac{3}{2 \mu_{x}^{-1}+\mu_{z}^{-1}} \frac{e^{4}}{2 \hbar^{2} \kappa^{2}}
$$

are given in Fig. 1 as a function of $\gamma^{1 / 3}$. It is seen here that for a constant value of the trace of $\boldsymbol{u}^{-1}$ the groundstate energy is varying less than $5 \%$ in the range $0.5<\gamma<2$. Based on this fact we have assumed that the case $\mu_{x} \neq \mu_{y} \neq \mu_{z}$ can be calculated with reasonable accuracy, if two of the masses, say $\mu_{x}$ and $\mu_{y}$, differ by less than a factor of 2 , by using the curve in Fig. 1 with $\mu_{x}{ }^{-1}$ and $\mu_{y}{ }^{-1}$ replaced by their mean value.

We shall now use the above assumptions and the results in Fig. 1 to calculate the high-stress exciton binding energies associated with each of the band pairs of the split indirect energy gap.

The conduction-band contribution to $\boldsymbol{u}^{-1}$ of the relative motion is characterized by a longitudinal mass $m_{L}$ and a transverse mass $m_{T}$. For [001] and [111] stress the hole energy surfaces near $\mathbf{k}=0$ are two ellipsoids, one prolate and one oblate, both having symmetry about the stress axis. The hole masses $m_{11}$ and $m_{\perp}$ parallel and perpendicular to the stress are given by ${ }^{19}$

$$
\begin{aligned}
\frac{1}{m_{11}}=A \pm B & \text { for }[001] \text { stress } \\
=A \pm \frac{1}{3} N & \text { for }[111] \text { stress } \\
\frac{1}{m_{\perp}}=A \mp \frac{1}{2} B & \text { for }[001] \text { stress } \\
=A \mp \frac{1}{6} N & \text { for }[111] \text { stress. }
\end{aligned}
$$

Inserting these masses for electrons and holes in Eq. (4.1) it is seen that

$$
\operatorname{Tr}\left(\mathfrak{u}^{-1}\right)=\operatorname{Tr}\left(\mathbf{m}_{v}^{-1}\right)+\operatorname{Tr}\left(\mathbf{m}_{c}^{-1}\right)=3 A+m_{L}^{-1}+2 m_{T^{-1}}
$$

is independent of the valence band considered. Thus, the value of $E_{0}$ of Eq. (4.3) is the same for any combination of band pairs in the strained crystal. Different values of $E_{\text {ex }}$ arise from the fact that the degree of anisotropy of $\mathfrak{u}^{-1}$ depends on the valence band in question and on the angle between the symmetry directions of the valence and conduction ellipsoids.

In Table III ${ }^{20,21}$ the approximate values of $E_{\text {ex }}$ are listed for the band pairs denoted by $i, j$ in Eq. (2.7). The deviation of the results from the isotropic value is seen to be small except for [111] stress in Ge. Here, the

${ }^{18}$ R. W. Keyes, IBM J. Res. Develop. 5, 65 (1961).

${ }^{19}$ The mass shift with stress in Si (see Ref. 11) is not included in these expressions. The contribution from this to the exciton energy shifts has been found to be small compared to the experimental uncertainty.

${ }_{20}^{20}$ B. Levinger and D. Frankl, J. Phys. Chem. Solids 20, 281 (1961).

${ }^{21}$ R. N. Dexter, B. Lax, A. F. Kip, and G. Dresselhaus, Phys. Rev. 96, 1222 (1954). 
TABLE III. Calculated exciton energies in meV for strained silicon and germanium. The indicated shifts $\Delta E_{\mathrm{ex}}$ are relative to the calculated zero-stress values $12.7 \mathrm{meV}$ (Si) and $3.0 \mathrm{meV}(\mathrm{Ge})$ of Ref. 15. The effective mass parameters of Refs. 11, 14, 20, and 21 and the dielectric constants $12.0 \mathrm{(Si}$ ) and $16.0(\mathrm{Ge})$ has been used in the calculations. For comparison it should be mentioned that the results $12.0 \mathrm{meV}$ for $\mathrm{Si}$ and $2.5 \mathrm{meV}$ for Ge are found in case of isotropic reduced mass equato to $\frac{1}{3} m_{L}+\frac{2}{3} m_{T}+A$.

\begin{tabular}{|c|c|c|c|c|c|c|c|c|c|}
\hline \multirow{3}{*}{\multicolumn{2}{|c|}{$\begin{array}{c}\text { Material } \\
\text { Valley } \\
\text { direction } \\
\text { Shape of valence- } \\
\text { band ellipsoid }\end{array}$}} & \multicolumn{4}{|c|}{$\mathrm{Si}$} & \multicolumn{4}{|c|}{$\mathrm{Ge}$} \\
\hline & & \multicolumn{2}{|c|}{$[001],[001]$} & \multicolumn{2}{|c|}{$\begin{array}{l}{[100],[010]} \\
{[\overline{1} 00],[0 \overline{1} 0]}\end{array}$} & \multicolumn{2}{|c|}{ [111] } & \multicolumn{2}{|c|}{$\begin{array}{c}{[11 \overline{1}],[1 \overline{1} 1]} \\
{[\overline{1} 11]}\end{array}$} \\
\hline & & prolate & oblate & prolate & oblate & prolate & oblate & prolate & oblate \\
\hline$[001]$ stress & $\begin{array}{r}E_{\text {ex }} \\
\Delta E_{\text {ex }}\end{array}$ & $\begin{array}{r}12.3 \\
-0.4\end{array}$ & $\begin{array}{r}12.0 \\
-0.7\end{array}$ & $\begin{array}{r}12.4 \\
-0.3\end{array}$ & $\begin{array}{r}12.1 \\
-0.6\end{array}$ & $\begin{array}{r}2.6 \\
-0.4\end{array}$ & $\begin{array}{r}2.5 \\
-0.5\end{array}$ & $\begin{array}{r}2.6 \\
-0.4\end{array}$ & $\begin{array}{r}2.5 \\
-0.5\end{array}$ \\
\hline$[111]$ stress & $\begin{array}{r}E_{\text {ex }} \\
\Delta E_{\text {ex }}\end{array}$ & $\begin{array}{r}12.5 \\
-0.2\end{array}$ & $\begin{array}{r}12.0 \\
-0.4\end{array}$ & $\begin{array}{r}12.5 \\
-0.2\end{array}$ & $\begin{array}{r}12.0 \\
-0.4\end{array}$ & $\begin{array}{r}3.4 \\
+0.4\end{array}$ & $\begin{array}{r}2.5 \\
-0.5\end{array}$ & $\begin{array}{r}2.5 \\
-0.5\end{array}$ & $\begin{array}{r}2.7 \\
-0.3\end{array}$ \\
\hline
\end{tabular}

anisotropy associated with the prolate valence band and the $[111]$ valley is characterized by $\gamma=0.1$ which leads to $30 \%$ increase in the binding energy.

\section{THE INDIRECT OPTICAL ABSORPTION EDGE}

A detailed presentation of the theory and the experimental results of the indirect absorption edge in silicon and germanium is given by McLean. ${ }^{22}$ The theory is based on a second-order perturbation calculation in which the radiation and the lattice vibrations are acting on the electronic system.

The spectral dependence of the absorption contribution from one type of phonon interaction can be characterized by a threshold energy given by

$$
E_{t} \equiv E_{g}-E_{\mathrm{ex}} \pm E_{p}
$$

where $E_{g}$ is the indirect energy gap and $E_{\text {ex }}$ and $E_{p}$ are the binding energy of a ground-state exciton and the energy of the phonon both with wave vector equal to that of the conduction minimum. \pm correspond to phonon emission or absorption. For photon energies larger than $E_{t}$ the absorption in the allowed case is rising as the square root of the excess energy due to creation of bound excitons whereas a $\frac{3}{2}$ power absorption producing unbound excitons will have a threshold at $E_{t}+E_{\text {ex }}$. In the forbidden case the corresponding power laws are $\frac{3}{2}$ and $\frac{5}{2}$.

In the unstrained case the transitions are specified by creation of a bound or an unbound exciton and creation or destruction of a phonon. Under the application of shear stress we must also specify which valence band and which conduction valley are involved in the process. The contribution from each of these types of transitions can then be treated using the same methods as previously.

These contributions are first of all characterized by the individual threshold energies given by

$$
E_{t}^{i j}=E_{o}^{i j}-E_{\mathrm{ex}}^{i j} \pm E_{p}{ }^{i} .
$$

${ }^{22}$ T. P. McLean, Progress in Semiconductors, edited by A. F. Gibson (Heywood and Company, London, 1960), Vol. 5.
These three terms have been discussed in the previous sections. Important as well for the interpretation of the present experiments are the relative magnitudes, the polarization dependence and the spectral behavior of each of the transitions denoted by $i, j$ in Eq. (5.2). We shall return to these features of the strain-induced fine structure of the indirect edge in the discussion of the experimental data.

\section{THE EXPERIMENTAL PROCEDURE}

Optics

In the experimental investigation of a splitting phenomenon it is desirable to determine the individual energy levels as well as the shapes of the split components. Such an analysis of a threshold for a square-root rise of the optical absorption requires very high stability of light source, detector, and electronics when the investigation is carried out in a conventional spectrom-

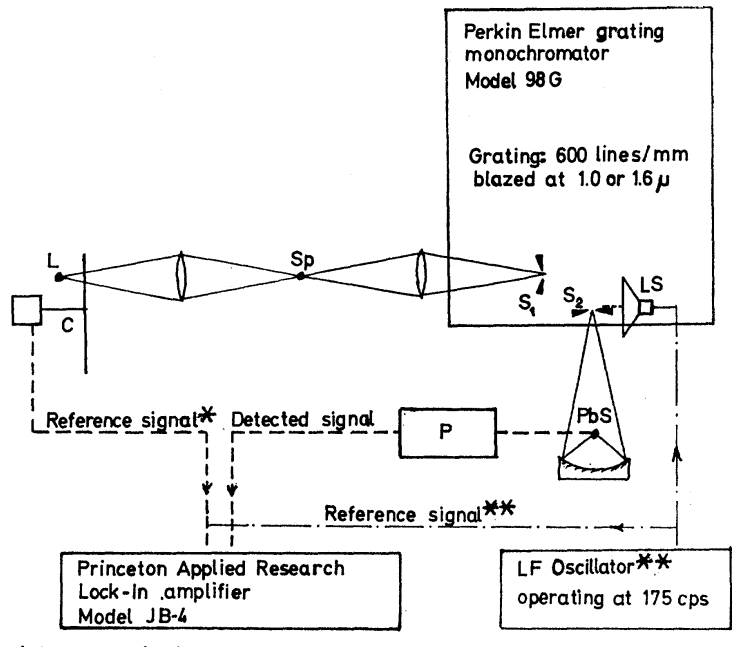

*) for conventional operation

$\left.{ }^{* x}\right)$ for wavelength modulation

Fig. 2. The optical set-up used in the experiments. L: Light source; C: chopper for conventional operation; Sp: Specimen; $\mathrm{S}_{1} \mathrm{~S}_{2}$ : Slits; PbS: Lead sulphide detector; P: Preamplifier; and LS: loudspeaker for wavelength modulation. Single asterisk indicates "for conventional operation"; double asterisk indicates "for wavelength modulation." 
eter. Furthermore, the decomposition of the split threshold from the recorded transmission spectra is a complicated procedure in which processing of a large amount of data is necessary. These difficulties lead us to modify the traditional spectrometer as shown in Fig. 2.

Here, the chopper was removed and the exit slit $\left(S_{2}\right)$ was connected mechanically to a loudspeaker giving the slit a transverse vibrational movement. Thus, the conventional intensity modulation was replaced by a differential wavelength modulation. The oscillator frequency $(175 \mathrm{cps})$ applied to the loudspeaker was used as reference in the phase sensitive electronics. The signal detected in this way is proportional to

$$
\frac{s_{1} s_{2} s_{3}}{D^{2}\left(\lambda_{0}\right)}\left[\frac{d I(\lambda)}{d \lambda}\right]_{\lambda=\lambda_{0}}
$$

where $s_{1}$ and $s_{2}$ are widths of the slits $S_{1}$ and $S_{2}$ (see Fig. 1); $s_{3}$ is the peak to peak amplitude of the slit vibration; $I(\lambda)$ is the intensity for wavelength $\lambda$ of the light illuminating the entrance slit; $D\left(\lambda_{0}\right)$ and $\lambda_{0}$ are linear dispersion and wavelength setting of the monochromator. Here, we have assumed that the width of the line investigated is large compared to the conventional resolution $\frac{1}{2}\left(s_{1}+s_{2}\right) / D\left(\lambda_{0}\right)$. This is not the case in the measurements reported here but the errors introduced by insufficient instrumental resolution will not be severe in determining the splitting.

The resolution of the modified instrument is

$$
\left(s_{1}+s_{2}+s_{3}\right) / 2 D\left(\lambda_{0}\right),
$$

which means that the best choice for the slit widths and the vibration amplitude is $s_{1}=s_{2}=s_{3}$.

The intensity of the light falling on the detector is given by

$$
I(\lambda)=\frac{(1-R(\lambda))^{2} \exp (-\alpha(\lambda) d)}{1-R^{2}(\lambda) \exp (-2 \alpha(\lambda) d)} I_{0}(\lambda),
$$

where $\alpha, R$, and $d$ are absorption coefficient, reflectivity, and thickness of the sample and $I_{0}(\lambda)$ is the intensity without the sample. For our purpose we can put the denominator of Eq. (6.1) equal to unity and neglect the spectral dependence of $R$. If $d I_{0} / d \lambda$ is small compared to $d I / d \lambda$, one obtains

$$
d I / d \lambda=(1-R)^{2} I_{0} \exp (-\alpha(\lambda) d)(d \alpha(\lambda) / d \lambda) .
$$

This implies that the vibrating slit spectrometer essentially is measuring $d \alpha / d \lambda$ reduced by the factor $\exp (-\alpha d)$.

However, if the contribution to $d I / d \lambda$ from the source spectrum and atmospheric absorption is comparable to that of the sample, the derivative of Eq. (6.1) has a more complicated form. In this case a separate determination of $d I_{0} / d \lambda$ is necessary and the measured transmission spectra for the sample should be corrected accordingly.

The application of the experimental technique using

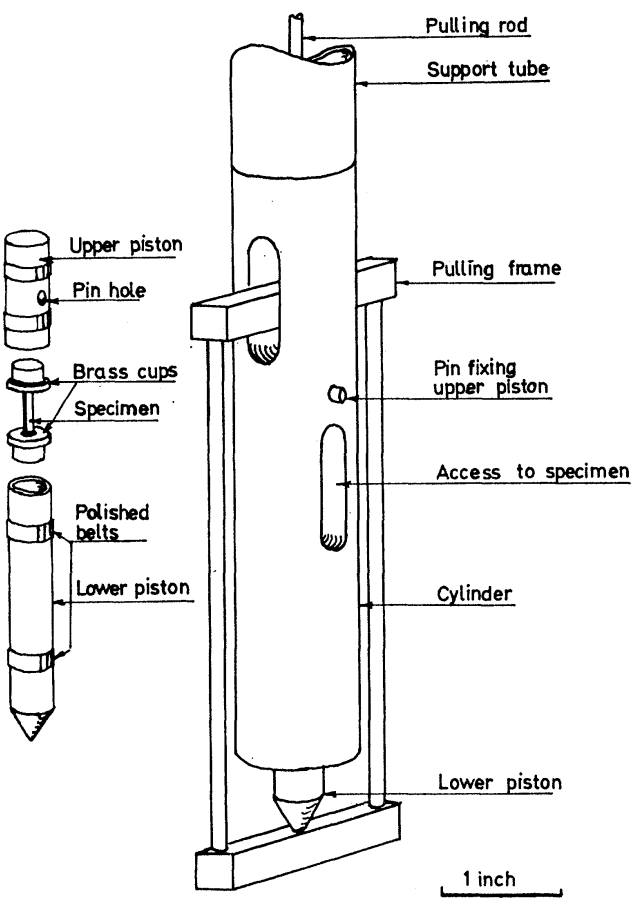

Frg. 3. Strain apparatus for low-temperature operation. Suitable for compressive forces up to $500 \mathrm{~kg}$ and for cross sections between 1 and $10 \mathrm{~mm}^{2}$.

a vibrating slit in the spectrometer has first been described in a recent note by the author. ${ }^{25}$ It is believed that this and analogous techniques for obtaining a differential wavelength modulation can be used to advantage also in other types of optical experiments, e.g., in the investigation of the weak structure in reflection spectra. ${ }^{23}$

\section{Cooling and Straining Technique}

The high resistivity $\mathrm{Si}(900 \Omega \mathrm{cm})$ and $\mathrm{Ge}(40 \Omega \mathrm{cm})$ single crystals were $x$-ray oriented in the desired direction within $1^{\circ}$ and strained in the apparatus shown in Fig. 3. This set-up is similar to that used by Fritzsche. ${ }^{24}$ The parts were made of stainless steel, and the inside of the cylinder as well as the belts on the pistons were polished and fitted together. A force applied upwards to the pulling rod is transferred through the frame to the lower piston giving the sample a compressive stress. The length of the thin-walled support tube and the pulling rod ( 7 in.) allowed the apparatus to be operated at low temperatures. The sample was cemented into holes in the brass cups by means of an epoxy glue and its dimensions were $15 \mathrm{~mm}$ in length and cross sections between $1 \times 1 \mathrm{~mm}^{2}$ and $5 \times 4 \mathrm{~mm}^{2}$.

Low-temperature measurements were carried out in an unsilvered glass Dewar containing liquid nitrogen at

${ }^{23}$ H. R. Philipp, W. C. Dash, and H. Ehrenreich, Phys. Rev. 127, 762 (1962).

${ }^{24}$ M. Cuevas and H. Fritzsche, Phys. Rev. 137, A1847 (1965). 
a level a few centimeters below the sample. A tightfitting copper tube surrounding the entire cylinder body acted as a thermal contact to the upper part of the strain apparatus. This technique yielded sample temperatures of $80^{\circ} \mathrm{K}$ and frequency adjustments of the nitrogen level enabled us to keep the temperature drift within $\pm 1^{\circ} \mathrm{K}$.

\section{EXPERIMENTAL RESULTS}

Directly detected spectra of $d I / d \lambda$ for strained and unstrained $\mathrm{Si}$ at $80^{\circ} \mathrm{K}$ are shown in Figs. 4, 5, and 6 corresponding to the region around the energy threshold $1.210 \mathrm{eV}$ specified by emission of a TO phonon. The instrumental resolution including the contribution from the vibrating slit was $1.2 \mathrm{MeV}$. Preliminary data similar to these have been reported by the author. ${ }^{25}$ The square-root contribution in the unstrained crystal is seen to appear as a peak corresponding to a broadened

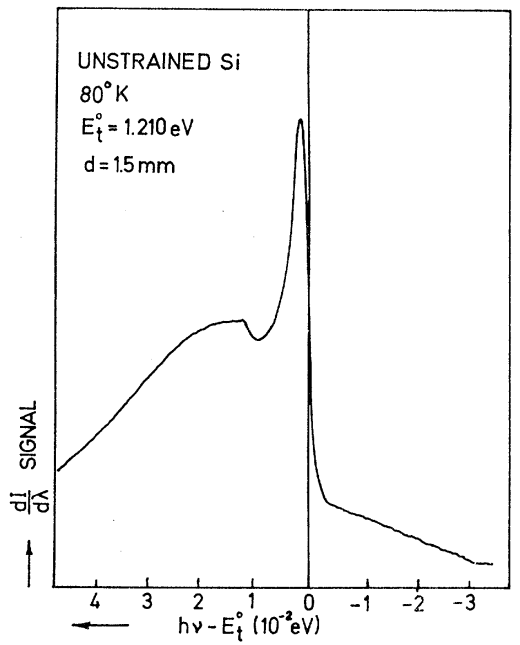

FIG. 4. Recorded transmission spectra of $d I / d \lambda$ for silicon at $80^{\circ} \mathrm{K}$. Stress direction and magnitude, photon energy range, and thickness $d$ of the sample are indicated.

$-\frac{1}{2}$ power function. For higher photon energies the $\frac{3}{2}$ power absorption show up in $d I / d \lambda$ as a square-root curve reduced by the factor $\exp (-\alpha d)$. The steepness of the low-energy rise of the peak is a measure of the Gaussian broadening and/or the instrumental resolution. The observed broadening characterized by the half-width can be estimated to be $2.5 \mathrm{meV}$ in Fig. 4 . For moderate [001] stress (Fig. 5) the single peak splits up into four components with contributions to the absorption of magnitude $\alpha_{i}{ }^{001}(i=1,2,3,4)$ where increasing $i$ refers to increasing threshold energy. For higher magnitudes of stress the broadening of $\alpha_{2}{ }^{001}$ and $\alpha_{4}{ }^{001}$ reaches values of the order of 10 to $15 \mathrm{meV}$ whereas the peaks from $\alpha_{1}{ }^{001}$ and $\alpha_{3}{ }^{001}$ remain sharp. For [111] stress (Fig. 6) none of the two peaks $\alpha_{1}^{111}$ and $\alpha_{2}^{111}$ observed is broadening considerably even for very high values of stress. Spectra for strain in the $[110]$ direction yielded three peaks and a pronounced broaden-

${ }^{25}$ I. Balslev, Solid State Comm. 3, 213 (1965).

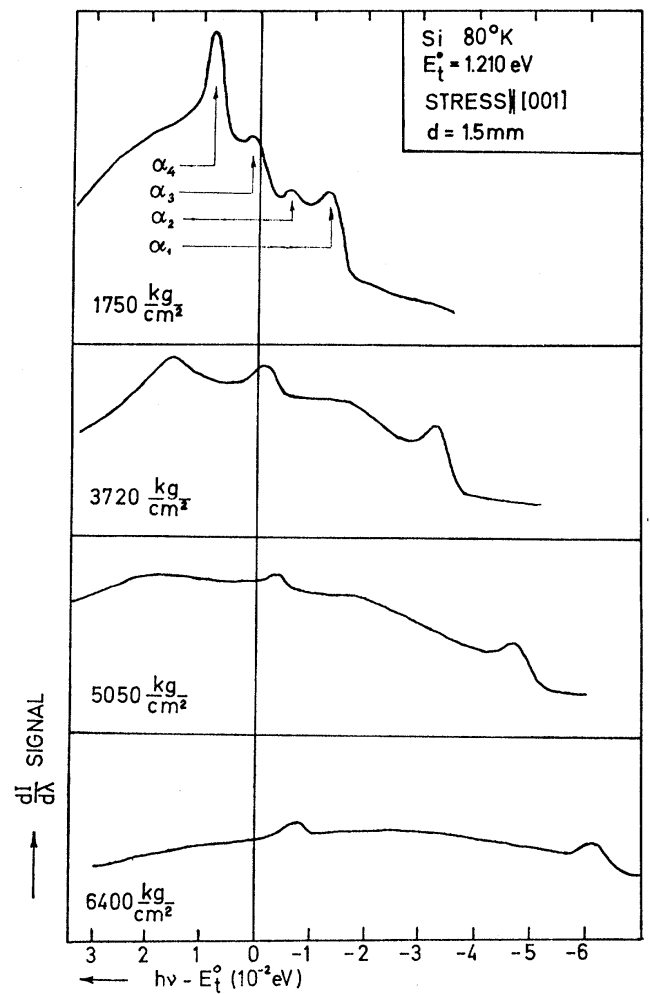

FIG. 5. Recorded transmission spectra of $d I / d \lambda$ for silicon at $80^{\circ} \mathrm{K}$. Stress direction and magnitude, photon energy range, and thickness $d$ of the sample are indicated.

ing for the high-energy peak. Defining the thresholds as the low-energy half points of the peaks we have plotted the splitting in Fig. 7. The straight lines in Fig. 7 and similar lines for [110] stress yielded the slope values and

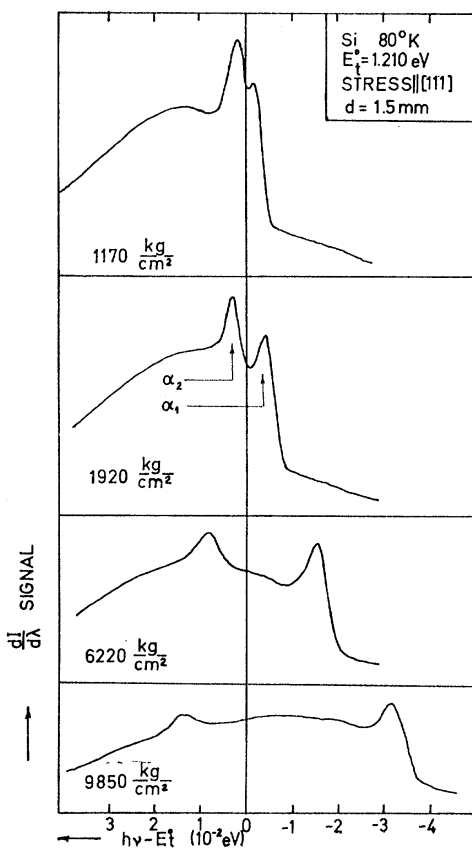

FIG. 6. Recorded transmission spectra of $d I / d \lambda$ for silicon at $80^{\circ} \mathrm{K}$. Stress direction and magnitude, photon energy range, and thickness $d$ of the sample are indicated. 
TABLE IV. Slopes and extrapolated zero-stress levels obtained from the observed splitting. The uncertainty of the determination of the slopes corresponds to approximately $10^{-7} \mathrm{eV} \mathrm{cm} / \mathrm{kg}$. The results of the extrapolation are given relative to the true zero-stress threshold energy. In terms of shifts in the exciton binding energy the sign of these results should be reversed.

\begin{tabular}{|c|c|c|c|c|c|c|c|c|}
\hline \multirow{2}{*}{$\begin{array}{l}\text { Component } \\
{ }^{\circ} \mathrm{K}\end{array}$} & \multicolumn{4}{|c|}{ Slopes in units of $10^{-6} \mathrm{eV} \mathrm{cm} / \mathrm{kg}$} & \multicolumn{4}{|c|}{ Extrapolated zero-stress threshold energies in $\mathrm{meV}$} \\
\hline & $\alpha_{1}$ & $\alpha_{2}$ & $\alpha_{3}$ & $\alpha_{4}$ & $\alpha_{1}$ & $\alpha_{2}$ & $\alpha_{3}$ & $\alpha_{\mathbf{l}}$ \\
\hline [001] stress $\mathrm{Si}, 80$ & -9.3 & $-4.6^{\mathrm{a}}$ & -1.0 & $+3.7^{\mathrm{a}}$ & $+1.0 \pm 0.5$ & $0.0 \pm 0.5$ & $+1.0 \pm 0.5$ & $0.0 \pm 0.5$ \\
\hline [111] stress $\mathrm{Si}, 80$ & -3.0 & +0.7 & $\ldots$ & $\ldots$ & $+1.0 \pm 0.5$ & $0.0 \pm 0.5$ & $\ldots$ & $\ldots$ \\
\hline$[110]$ stress $\mathrm{Si}, 80$ & -4.6 & \multicolumn{2}{|c|}{-0.8} & $+3.4^{\mathrm{a}}$ & \multirow{2}{*}{\multicolumn{4}{|c|}{$\begin{array}{r}+1.0 \pm 0.5 \\
0.0 \pm 1.0\end{array}$}} \\
\hline$[001]$ stress Si, 295 & -9.2 & $-4.7^{\mathrm{a}}$ & -0.4 & $+4.3^{\mathrm{a}}$ & & & & \\
\hline$[001]$ stress $\mathrm{Ge}, 80$ & -1.7 & $+2.1^{b}$ & $\ldots$ & $\ldots$ & $+1.0 \pm 0.2$ & $+0.8 \pm 0.5$ & $\ldots$ & $\ldots$ \\
\hline$[111]$ stress $\mathrm{Ge}, 80$ & -7.8 & $-4.8^{\mathrm{a}}$ & +2.8 & $+5.2^{\mathrm{a}}$ & $+0.6 \pm 0.2$ & $-0.3 \pm 0.3$ & $+0.4 \pm 0.2$ & $+0.8 \pm 0.2$ \\
\hline$[110]$ stress $\mathrm{Ge}, 80$ & -4.4 & $-1.2^{\mathrm{a}}$ & +3.1 & $+6.4^{\mathrm{a}}$ & \multicolumn{4}{|c|}{$+0.7 \pm 0.3$} \\
\hline
\end{tabular}

a Broadening with increasing stress.

b Uncertain result due to very pronounced broadening.

the extrapolated zero stress energies given in Table IV.

In $\mathrm{Si}$ at room temperature the two thresholds at 1.169 and $1.053 \mathrm{eV}$ for emission and absorption of a TO phonon were examined in the presence of [100] stress. Four peaks as at $80^{\circ} \mathrm{K}$ were observed although the increased broadening lead to a more uncertain quantitative analysis of the splitting. The linear dependence of the four levels given in Table IV was found to be the same for the two thresholds within the experimental accuracy.

The spectra for Ge at $80^{\circ} \mathrm{K}$ shown in Figs. 8 and 9 were recorded near the $0.761 \mathrm{eV}$ threshold from emission

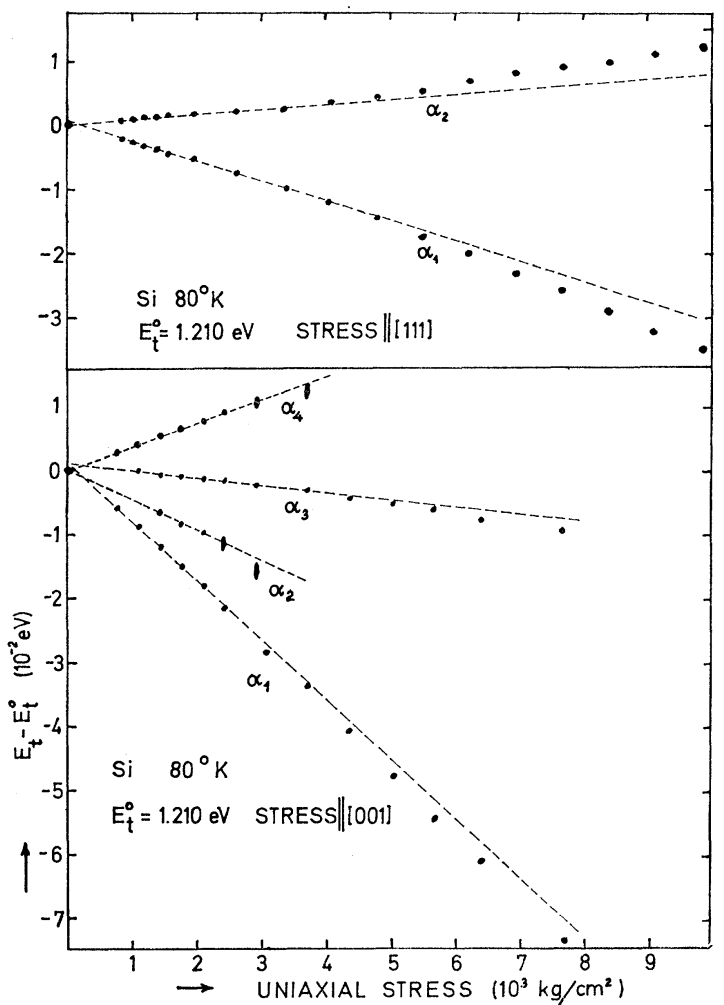

FIG. 7. Observed threshold energies $E_{t}$ versus stress for $\mathrm{Si}$ at $80^{\circ} \mathrm{K}$ stressed in the [111] and [001] directions. of a longitudinal acoustic (LA) phonon. The instrumental resolution was $0.5 \mathrm{meV}$. In the spectra for the unstrained crystals the broadening is $0.7 \mathrm{meV}$ and the additional structure on the high-energy side of the main peak indicates allowed transitions with thresholds $8.2 \pm 0.2$ and $4.0 \pm 0.3 \mathrm{meV}$ above the LA threshold. Apart from energy scale the features of the splitting in [111] and [110] strained germanium are similar to that of $[001]$ strained silicon. The broadening of the peaks from $\alpha_{2}{ }^{111}, \alpha_{4}{ }^{111}, \alpha_{2}{ }^{110}$, and $\alpha_{4}{ }^{110}$ reaches values of the order of 3 to $5 \mathrm{meV}$ for a stress of $2000 \mathrm{~kg} / \mathrm{cm}^{2}$. In contrast to $\alpha_{2}{ }^{111}$ in Si one of the two peaks observed for [001] strained Ge is broadening with increasing stress. The observed threshold energies are plotted versus stress in Fig. 10 and the slopes of the splitting lines and extrapolated zero stress energies are given in Table IV.

The relative magnitudes of the peaks were found to depend on the polarization direction. The results of recorded spectra using light polarized parallel and perpendicular to the stress axis are summarized in Table $\mathrm{V}$ giving the approximate fractional strengths of the contributions $\alpha_{i}$.

'Finally, it should be mentioned that the wavelength behavior of the light detected without sample was

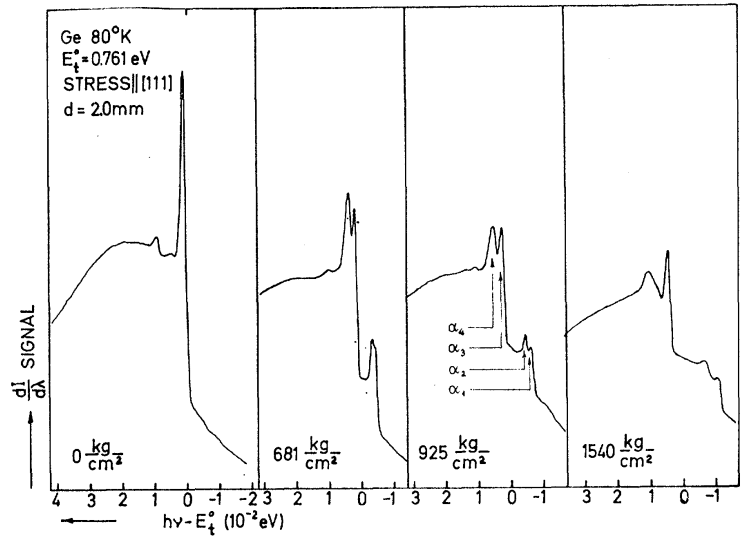

FIG. 8. Recorded transmission spectra of $d I / d \lambda$ for germanium at $80^{\circ} \mathrm{K}$. Stress direction and magnitude, photon energy range, and sample thickness $d$ are indicated. 
TABLE V. Observed fractional contributions at $80^{\circ} \mathrm{K}$ of the split components $\alpha_{i}$ for various stress and polarization directions.

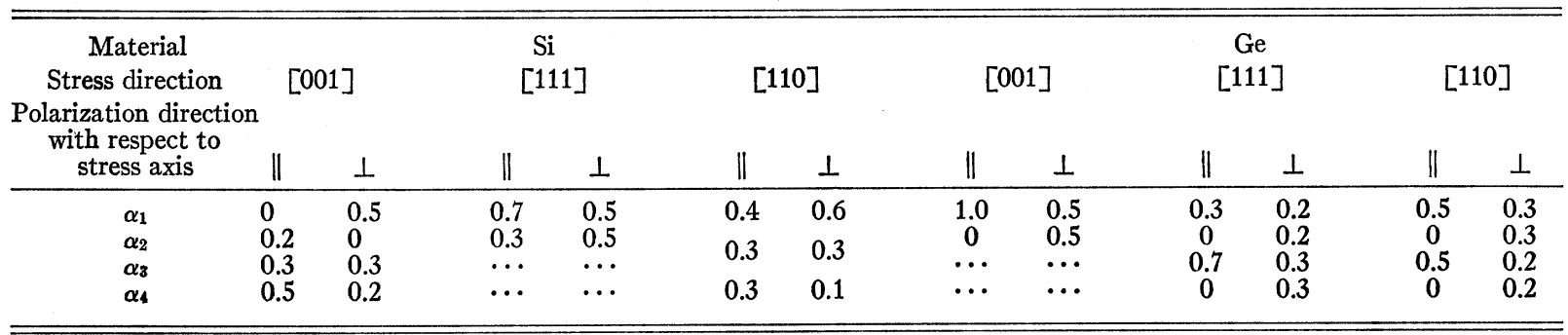

sufficiently smooth for Eq. (6.2) to be valid near all the thresholds investigated.

\section{INTERPRETATION AND DISCUSSION}

The interpretation of the data is based on the neglect of the phonon contribution to the splitting and on the assumption that the exciton binding energy is stressindependent for high magnitudes of stress. Thus, the straight lines in Figs. 6 and 9 can be related directly to Eq. (2.7) and to Tables I and II. The assignment of the split components can be obtained by comparing the three stress directions. Then, the slope values of the components and appropriate elastic constants ${ }^{26}$ will yield results for the quantities $\Xi_{u},|b|,|d|$, and $\Xi_{\boldsymbol{d}}$ $+\frac{1}{3} \Xi_{u}-a$. Information on the high-stress exciton energies relative to the zero-stress value can be derived by extrapolation to zero stress of the splitting lines.

In the following we shall discuss the information which can be gained by application of the above procedure and by examining the polarization dependence of the splitting. Although the features of the unstrained crystals have been discussed thoroughly by $M M Q R, 12$ we shall make a few remarks on additional information derived from our spectra of strain-free crystals.

\section{Silicon}

The spectra for unstrained $\mathrm{Si}$ at $80^{\circ} \mathrm{K}$ (Fig. 4) indicate that the observed broadening is mainly due to

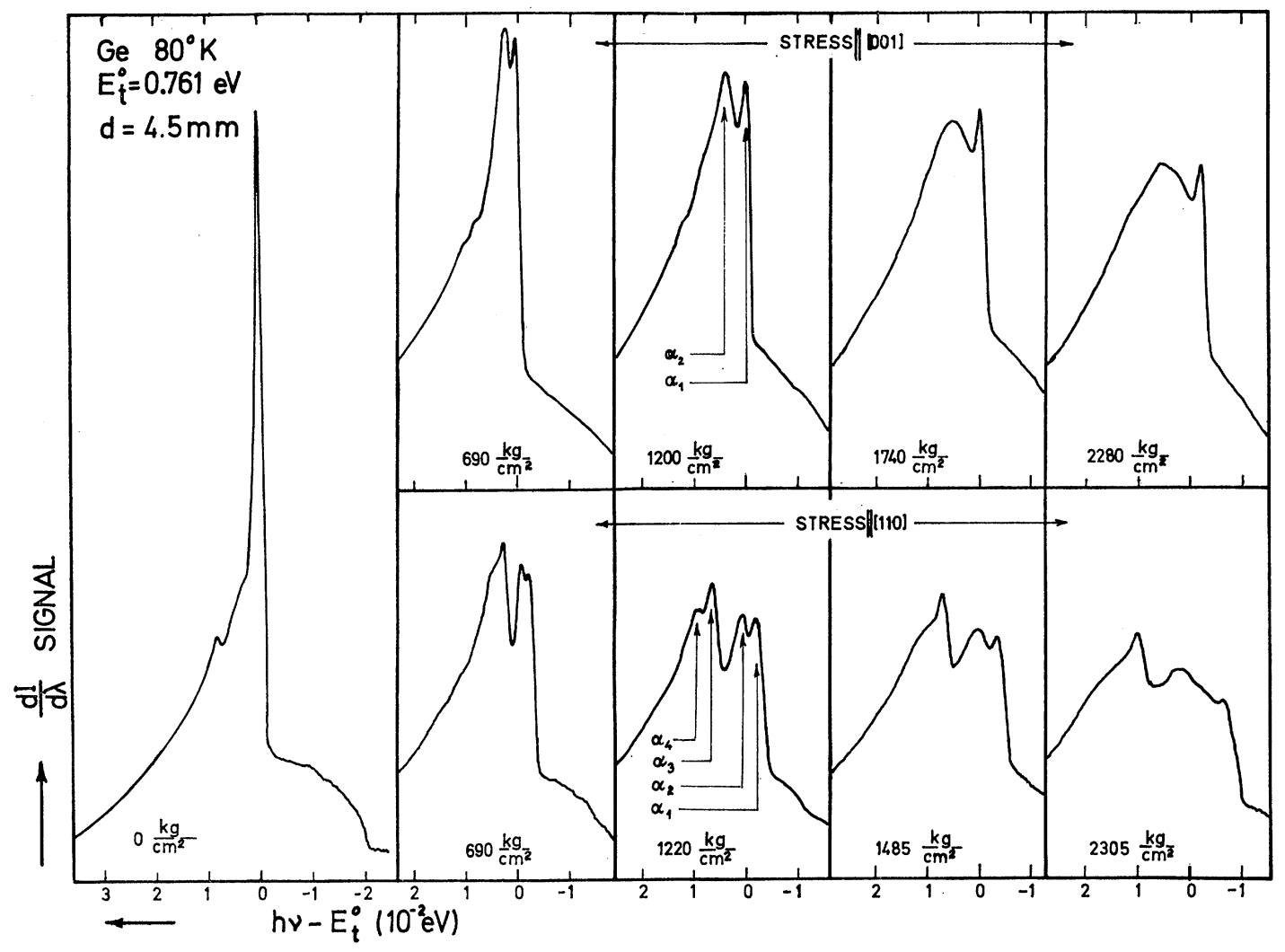

FIg. 9. Recorded transmission spectra of $d I / d \lambda$ for germanium at $80^{\circ} \mathrm{K}$. Stress direction and magnitude, photon energy range, and sample thickness $d$ are indicated.

${ }^{26}$ H. J. McSkimin, J. Appl. Phys. 24, 988 (1953). 


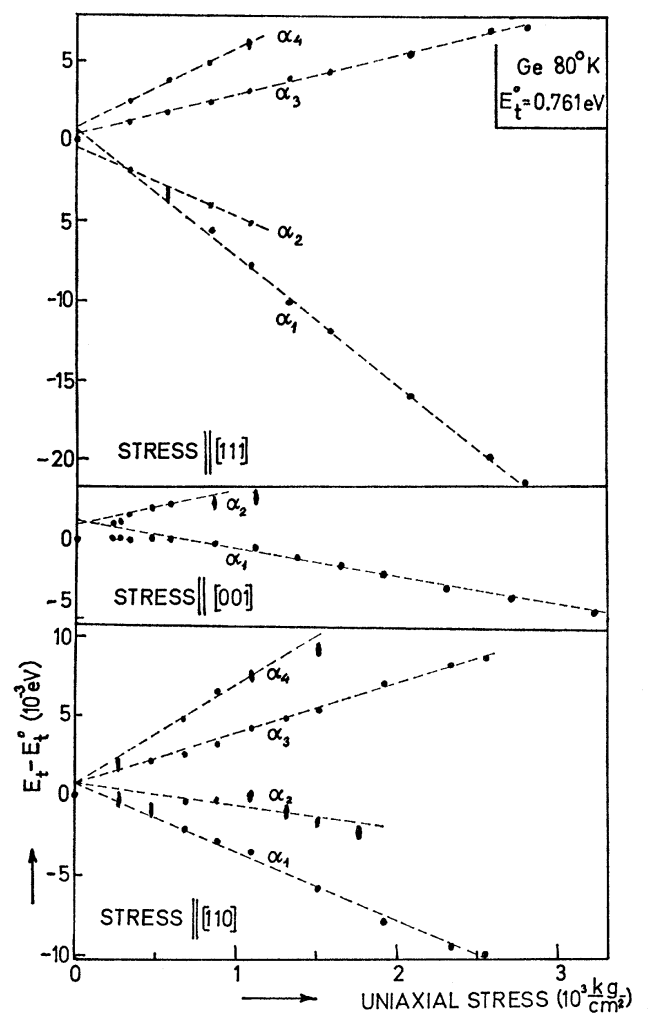

FIG. 10. Observed threshold energies $E_{t}$ versus stress for germanium at $80^{\circ} \mathrm{K}$.

our limited instrumental resolution. Our broadening of $2.5 \mathrm{meV}$ should be compared with $1.2 \mathrm{meV}$ observed by MMQR. ${ }^{12}$ The unexplained, previously reported square root rise in the absorption $5.5 \mathrm{meV}$ above the principal threshold ${ }^{22}$ is not presented in our spectra. It is evident that this deviation of our data from the previous results cannot be explained by insufficient resolution because strain-induced splittings less than $5 \mathrm{meV}$ were resolved in our apparatus. The threshold for the $\frac{3}{2}$ power absorption is found to be $10 \pm 2 \mathrm{meV}$ above the exciton ground-state level, which is in agreement with Ref. 22.

The number of peaks in the spectra for the three stress directions agrees with the accepted position in the reduced zone of the conduction-band valleys. The presence of only three peaks for [110] stress indicates approximately equal splitting of valence and conduction band.

The assignment of the split components $\alpha_{1}^{111} \alpha_{2}^{111}$, $\alpha_{1}{ }^{001}$, etc., and the slope values in Table IV lead to the deformation potentials quoted in Table $\mathrm{VI} .^{27}$ The consistency for the three stress directions is seen to be satisfactory.

${ }^{27}$ The difference between these values and those of the previously published preliminary measurements (Ref. 25) is due to a more detailed analysis of the low-stress region with increased resolution and to a revised interpretation.
TABLE VI. Deformation potentials in eV derived from Table IV and from elastic constants of Ref. 26. The values for $\Xi_{u}$ were calculated from the slope difference of the components $\alpha_{1}$ and $\alpha_{3}$. Two results for $|b|$ or $|d|$ in some of the columns correspond to the pairs $\alpha_{1} \alpha_{2}$ and $\alpha_{3} \alpha_{4}$. For [110] stress the values of $d$ are obtained by putting $|b|=2.4 \mathrm{eV}$ and $1.6 \mathrm{eV}$ for $\mathrm{Si}$ and $\mathrm{Ge}$, respectively.

\begin{tabular}{|c|c|c|c|c|c|c|c|}
\hline \multirow{2}{*}{$\begin{array}{c}\text { Material } \\
\text { Temperature } \\
\text { Stress } \\
\text { direction } \\
\end{array}$} & \multirow[b]{2}{*}{ [001] } & \multicolumn{2}{|c|}{$80^{\circ} \mathrm{K}$} & \multicolumn{2}{|l|}{$295^{\circ} \mathrm{K}$} & \multicolumn{2}{|c|}{$\begin{array}{c}\mathrm{Ge} \\
80^{\circ} \mathrm{K}\end{array}$} \\
\hline & & [111] & {$[110]^{\mathrm{a}}$} & [001] & [001] & [111] & [110] \\
\hline$\Xi_{u}$ & +8.6 & $\cdots$ & +8.0 & +9.2 & $\cdots$ & +16.6 & $\pm \mathbf{1 5 . 9}$ \\
\hline$|b|$ & $\begin{array}{l}2.4 \\
2.4\end{array}$ & $\cdots$ & $\cdots$ & $\begin{array}{l}2.3 \\
2.0\end{array}$ & 1.6 & $\cdots$ & $\cdots$ \\
\hline$|d|$ & $\cdots$ & 5.3 & $\begin{array}{l}4.7 \\
5.5\end{array}$ & $\cdots$ & $\cdots$ & $\begin{array}{l}3.6 \\
4.1\end{array}$ & $\begin{array}{l}3.6 \\
3.7\end{array}$ \\
\hline$\Xi_{\mathrm{d}}+\frac{1}{8} \Xi_{u}-a$ & +4.3 & +3.4 & +4.3 & +3.1 & -0.5 & -2.4 & -2.2 \\
\hline
\end{tabular}

a Uncertain determination of splitting due to nearly equal slope of $\alpha_{2}$ and $\alpha_{8}$.

The nonlinear stress dependence of the $M_{J}= \pm \frac{1}{2}$ valence-band edge predicted by Hasegawa $\mathrm{a}^{5}$ [see Eq. (2.6)] should for negative signs of $b$ and $d$ (as obtained in Ref. 11) appear in Fig. 6 as negative quadratic contributions to the threshold energies of $\alpha_{1}{ }^{111}, \alpha_{1}{ }^{001}$, and $\alpha_{3}{ }^{001}$. This behavior is seen to be present (Fig. 7) and the magnitude of the quadratic shift is in agreement with the accepted value of the spin-orbit splitting $\Lambda=44$ $\mathrm{meV}$. However, the observed nonlinear behavior of $\alpha_{2}{ }^{111}$ for very high magnitudes of stress and the lack of broadening with stress of this component have not been explained.

The extrapolated zero-stress thresholds should be compared with the theoretical results in Table III. Although the uncertainty of the extrapolation is of the same order as the expected shifts in $E_{\text {ex }}$ the data seem to indicate that the upper valence band for compressive [001] and [111] stress is oblate. This leads to positive signs for the quantities $B b$ and $N d$.

The polarization dependence of the strengths of the individual transitions is due to the fact that the Hamiltonians for the two principal polarization directions of the light transform as two different representations of the noncubic group of the strained lattice. In $\mathrm{Si}$, no particular intermediate state is found to contribute strongly to the second-order matrix element for the indirect transitions. A group-theoretical investigation of the polarization dependence is therefore rather complicated. Consequently, we have not been able to make any safe identification of the split valence band in $\mathrm{Si}$ by analyzing the data given in Table $\mathrm{V}$ but the two zeroes for [001] stress may yield information on the sign of $b$.

Our resulting values and estimated accuracies for the deformation potentials $\Xi_{u},|b|$, and $|d|$ are listed in Table VII ${ }^{28,29}$ in which are also given the results from previous investigations. Our accuracies are experimental and do not include possible breakdown of the assumption concerning the phonon and exciton energies.

${ }^{28}$ H. Fritzsche, Phys. Rev. 115, 336 (1957)

${ }^{29}$ I. V. Dakhovskii, Fiz. Tverd. Tela 5, 2332 (1964) [English transl.: Soviet Phys.-Solid State 5, 1695 (1964)]. 
TABLE VII. Deformation potential constants from various experimental and theoretical methods. The experimental results are obtained for temperatures less than $100^{\circ} \mathrm{K}$.

\begin{tabular}{cccr}
\hline & Result of present work & Previous results & Ref. \\
\hline Silicon & & $7.7 \mathrm{eV}^{\mathrm{a}}$ & 6 \\
& & $8.3 \pm 0.3 \mathrm{eV}^{\mathrm{a}}$ & 7 \\
$\Xi_{u}$ & $\left(295^{\circ} \mathrm{K}: 9.2 \pm 0.3 \mathrm{eV}\right)$ & $11.3 \pm 1.3 \mathrm{eV}^{\mathrm{b}}$ & 10 \\
& & $9.5 \mathrm{eV}^{\mathrm{c}}$ & 30 \\
& & $1.4 \pm 0.15 \mathrm{eV}^{\mathrm{d}, \mathrm{e}}$ & 11 \\
$|b|$ & $2.4 \pm 0.2 \mathrm{eV}$ & $2.5 \mathrm{eV}^{\mathrm{c}, \mathrm{e}}$ & 30 \\
& & $7 \mathrm{eV}^{\mathrm{a}}$ & 8 \\
& & $3.1+0.3 \mathrm{eV}^{\mathrm{d}, \mathrm{e}}$ & 11 \\
$|d|$ & $5.3 \pm 0.4 \mathrm{eV}$ & $5.7 \mathrm{eV}^{\mathrm{c}, \mathrm{e}}$ & 30
\end{tabular}

Germanium

$\begin{array}{lllr} & & 17.3 \mathrm{eV}^{\mathrm{a}} & 6 \\ \Xi_{u} & 16.2 \pm 0.4 \mathrm{eV} & 19.2 \pm 0.4 \mathrm{eV}^{\mathrm{t}} & 28 \\ & & 18.9 \pm 1.7 \mathrm{eV}^{\mathrm{b}} & 10 \\ & & 16.5 \mathrm{eV}^{g} & 29 \\ & & 2.1 \pm 0.2 \mathrm{eV}^{\mathrm{t}} & 34 \\ |b| & 1.8 \pm 0.4 \mathrm{eV}^{\mathrm{e}} & 2.7 \pm 0.3 \mathrm{eV}^{\mathrm{b}} & 9 \\ & & 7.0 \mathrm{eV}^{\mathrm{f}} & 34 \\ & & 4.7 \pm 0.5 \mathrm{eV}^{\mathrm{b}} & 9\end{array}$

a Piezoresistance.

b Free-carrier piezobirefringence.

- Band calculations.

d Cyclotron resonance.

Sign has been determined as negative.

Studies of acceptor and donor binding energies.

b Splitting in the direct

Our magnitudes of $b$ and $d$ for silicon agree fairly well with the results from band calculations ${ }^{30}$ but they are approximately $50 \%$ higher than the results obtained from cyclotron-resonance experiments. ${ }^{11}$ However, recent estimates ${ }^{31}$ of some higher order terms in the theory of Hasegawa ${ }^{5}$ previously neglected in the application to the observed hole mass shift ${ }^{11}$ indicated corrections bringing the cyclotron-resonance results into agreement with those of the present work. As for the conduction-band deformation potential $\Xi_{u}$, the agreement between the results obtained by the different methods is seen to be fairly good.

In the room-temperature measurements on $\mathrm{Si}$ the values obtained for $\Xi_{u}$ were the same for the two thresholds corresponding to absorption and emission of the same phonon (TO). This is a valuable check on the assumption concerning the negligible contribution to the splitting from the phonon energy shifts. Another interesting result derived from the room-temperature data is the 6 to $8 \%$ increase of the $\Xi_{u}$ value compared to the value of $80^{\circ} \mathrm{K}$. Safe conclusions on the temperature dependence of $\Xi_{u}$ have not been drawn previously.

Our value of $\Xi_{d}+\frac{1}{3} \Xi_{u}-a$ corresponds to the shift of

30 I. Goroff and L. Kleinman, Phys. Rev. 132, 1080 (1963).

${ }^{31}$ I. Balslev and P. Lawaetz, Phys. Letters 19, 6 (1965). the indirect gap with hydrostatic pressure equal to $d E / d p=(-3.8 \pm 0.5) \times 10^{-12} \mathrm{eV} \mathrm{cm} \mathrm{cm}^{2}$ dyne at $80^{\circ} \mathrm{K}$.

This value should be compared with the result -1.5 $\times 10^{-12} \mathrm{eV} \mathrm{cm} /$ dyne at $250^{\circ} \mathrm{C}$ obtained from pressure dependence of the intrinsic conductivity. ${ }^{32}$

\section{Germanium}

The observed broadening of $0.7 \mathrm{meV}$ for $\mathrm{Ge}$ at $80^{\circ} \mathrm{K}$ is twice that of Ref. 12 and the two exciton levels separated by $1.0 \mathrm{meV}$ have not been resolved in our spectra. These features of our data are a consequence of our modest resolution compared to that of Ref. 12 . The additional allowed transitions showing up in the recordings for unstrained Ge (Fig. 9) correspond to emission of phonons with temperatures $415^{\circ} \mathrm{K}$ (TO) and $365^{\circ} \mathrm{K}$ (TA). These transitions have not been observed previously but thresholds due to absorption of these phonons have been reported. ${ }^{22}$ The high temperatures required for detection of transitions corresponding to absorption of optical phonons prevented $\mathrm{MMQR}^{12}$ from determining whether these are allowed or forbidden. Our spectra established that both transitions are allowed.

The deformation potentials derived from the splitting in strained germanium are listed in Table VI. Here, the magnitude of $\left(\Xi_{d}+\frac{1}{3} \Xi_{u}-a\right)$ determining the mean shift of the energy gap is considerably smaller for [001] stress than for the other stress directions. This may be due to an incorrect determination of the slope of the strongly broadening $\alpha_{2}{ }^{001}$ component. If $|b|$ is calculated from the well-defined slope of $\alpha_{1}{ }^{001}$ and the mean shift of the [111] and [110] stress directions, one obtains $|b|=2.2 \mathrm{eV}$. This value, however, leads to inconsistency in the comparison of the valence-band splittings in case of [111] and [110] stress.

The extrapolation to zero stress of the four components for [111] stress (Fig. 9) yields exciton energies in the high-stress region which are in excellent agreement with the theoretical results in Table III provided the lower valence band is prolate, i.e., if $N d$ is positive. Because of uncertain determination of the splitting associated with $\alpha_{2}{ }^{001}$, our data do not yield a safe indication of the sign of $B b$.

An interesting feature of the splitting is that the region of stress for which the exciton binding energies change from zero-stress to high-stress values is between 0 and $500 \mathrm{~kg} / \mathrm{cm}^{2}$. At $500 \mathrm{~kg} / \mathrm{cm}^{2}$ the valence-band extrema are separated by 1.0 to $1.5 \mathrm{meV}$. A consequence of this is that the dominating valence-band Bloch functions appearing in the expansion for the exciton state $^{13}$ correspond to hole energies less than 1.0 to $1.5 \mathrm{meV}$ which is surprisingly low compared to the ground-state binding energy $(3 \mathrm{meV})$.

For Ge it has been established that the conduction

\footnotetext{
${ }^{32}$ W. Paul and G. L. Pearson, Phys. Rev. 98, 1755 (1955).
} 
band state at $\mathbf{k}=0$ with symmetry $\Gamma_{2}^{\prime}$ is the dominating intermediate state for the indirect transitions. ${ }^{22}$ In this case we are able to derive selection rules for the principal polarization directions of the light. For [001] strain the point group of the lattice ${ }^{33}$ is $D_{4 h}$ and the Bloch functions at $\mathbf{k}=0$ of the valence-band states transform as $M_{6}{ }^{+}$and $M_{7}{ }^{+}$for $M_{J}= \pm \frac{3}{2}$ and $\pm \frac{1}{2}$, respectively. The intermediate state including spin and [001] strain has the symmetry $M_{7}-$ and the radiation operator transforms as $M_{1}^{\prime}$ and $M_{5}^{\prime}$ for polarizations respectively parallel and perpendicular to the strain axis. The expansions

$$
M_{7}-\times M_{1}^{\prime}=M_{6}{ }^{+}+M_{7}{ }^{+}, \quad M_{7}-\times M_{5}{ }^{\prime}=M_{7}{ }^{+},
$$

indicate forbidden transitions from the $M_{J}= \pm \frac{3}{2}$ valence band. For polarization parallel to the [111] direction a similar selection rule is valid.

Experimentally, in case of stress parallel polarization the components due to transitions from the lower hole band are observed to be absent for any of the three stress directions (see Table V). Thus, the lower valence band for compressive strain is identified as the $M_{J}= \pm \frac{3}{2}$ band which leads to negative signs of $b$ and $d$.

The magnitudes of the deformation potentials and the estimated accuracies are listed in Table VII. It is seen here that our values and results from previous experiments (see Table VII) do not exhibit complete consistency. Thus, our value of $\Xi_{u}$ is slightly lower than the previous results. Apart from the probably uncertain result $|d|=7 \mathrm{eV}$ obtained by $\mathrm{Hall}^{34}$ the agreement for the valence-band parameters is fairly good.

Our data yield a rather uncertain determination of the shift of the energy gap with hydrostatic pressure. The value

$$
d E / d p=(+3 \pm 1) \times 10^{-12} \mathrm{eV} \mathrm{cm} / \mathrm{dyn} \text { at } 80^{\circ} \mathrm{K}
$$

derived from the data for [111] and [110] stress directions is considerably lower than the result $+8 \times 10^{-12}$ $\mathrm{eV} \mathrm{cm} /$ dyn at room temperature obtained from other experiments. ${ }^{35,36}$

\section{Broadening}

The broadening of the components referring to the lower valence band may be explained by arguments similar to those of McLean and Paige. ${ }^{37}$ The possibility

${ }^{33}$ G. F. Koster, Solid State Phys., 5, 173 (1957).

${ }^{34}$ J. J. Hall, Phys. Rev. 128, 68 (1962).

${ }^{35}$ D. M. Warschauer, W. Paul, and H. Brooks, Phys. Rev. 98, 1193 (1955).

${ }^{36}$ H. Y. Fan, M. L. Shephered, and W. Spitzer, Photoconductivity Conference, edited by R. G. Breckenridge, B. R. Russell, and E. E. Hahn, (John Wiley \& Sons, New York, 1956), p. 184.

${ }^{37}$ T. P. McLean and E. G. S. Paige, Proceedings of the Sixth International Conference on The Physics of Semiconductors, Exeter, 1962 (Institute of Physics and The Physical Society, London, 1962), p. 6. for a hole in the lower band being scattered by an acoustic phonon with wave vector close to zero into a state in the upper band gives rise to a decreased lifetime and therefore an increased broadening of peaks associated with the lower valence band. In the presence of conduction-band splitting a similar decrease of the lifetime of electrons created in the upper valley(s) was not observed. This is a consequence of the nonzero energy of phonons involved in the scattering of electrons from one valley to another.

\section{SUMMARY}

It is seen that the study of the fine structure due to uniaxial stress of the indirect absorption edge in silicon and germanium is a powerful method for exploring the band structure of strained crystals. The conclusions drawn have primarily been concerned with the shifts and the splitting phenomena of the band extrema leading to the determination of deformation potentials for both bands. The broadening of the indirect edge at $80^{\circ} \mathrm{K}$ and our limited instrumental resolution prevented us from analyzing in detail the shifts with shear strain of the exciton binding energy. In most of the cases the expected and observed exciton energy shifts were comparable to our experimental uncertainty. For [111] stress in Ge, however, we have been able to confirm the oblate and prolate nature of the hole energy surfaces in strained crystals through the studies of the high-stress exciton binding energies.

Finally, it should be mentioned that additional information concerning the excitons and the matrix elements for the indirect transitions may be gained by extended theoretical studies of the magnitude of the split components and by experiments at lower temperatures and with higher resolutions.

Note added in proof. Recently, independent investigations similar to these have been reported by Adler and Erlbach. ${ }^{38}$ Some discrepancies in the selection rules for Ge and in the experimental results have not been explained so far.

\section{ACKNOWLEDGMENTS}

The author would like to thank professor N. I. Meyer for his continued interest and encouragement in all phases of this work. Helpful discussions with P. Lawaetz and valuable experimental suggestions by T. Guldbrandsen are gratefully acknowledged. Thanks are due to Dr. H. Fritzsche for communicating information on his strain apparatus prior to publication.

\footnotetext{
${ }^{38}$ E. Adler and E. Erlbach, Phys. Rev. Letters 16, 87 (1966).
} 